\title{
Time spent in the goal box: Effects of frequency and schedule of reinforcement
}

JAMES ALLISON

INDIANA UNIVERSITY

Running times showed a typical partial reinforcement effect in a runway which allowed $S$ to retrace from goal to stem. Retrace times on nonreward trials agreed with frustration theory, in that (a) during training, partially rewarded $S s$ spent less time in the goal box than continuously nonrewarded $S$ s, and (b) during extinction, time spent in the goal box was a decreasing function of the frequency of prior reward.

The simple straight runway can be viewed as two mutually exclusive regions $i$, consisting of a start box and stem, and $\mathbf{j}$, consisting of a goal box. Its use has produced a vast experimental literature on the tendency to approach the goal region $\left(T_{i j}\right)$, but very little on $T_{j i}$, the tendency to leave the goal region (Adelman \& Maatsch, 1955; Brown, 1948; Lewis, 1964). In particular, $T_{j i}$ has not been studied in the context of partial reinforcement, although it bears on explanations of the partial reinforcement effect which refer to frustrative nonreward in $\mathbf{j}$ (Amsel, 1958).

To articulate $T_{j i}$ with frustration theory, it is assumed that on nonreward trials $T_{j i}$ reflects the aversiveness of $\mathbf{j}$. This assumption is supported by evidence that $T_{j i}$, as measured by strength of pull, is increased by previous experience with electric shock in $\mathrm{j}$ (Brown, 1948). According to frustration theory, frustration is an aversive event produced by nonreward in $j$, provided $S$ has previously experienced reward there, The intensity of frustration elicited by nonreward increases with the frequency of prior reward in $\mathbf{j}$. Consequently, on nonreward trials $\mathbf{T}_{\mathrm{ji}}$ should be greater following partial reward than continuous nonreward training. During extinction, $\mathrm{T}_{\mathrm{j} i}$ should be some increasing function of the frequency of prior reward in $j$.

The experiment reported here studied $T_{j i}$ by dispensing with the customary retrace door. Time in $\mathbf{i}$ (running time) and time in $\mathbf{j}$ (retrace time) were both treated as dependent variables during training and extinction. Since $T_{i j}$ is generally assumed to be a decreasing function of running time, $T_{j i}$ was assumed to be a decreasing function of retrace time. The predictions outlined above were investigated by varying the frequency $(0,25$, or 50$)$ and the percentage $(0,50$, or 100) of training trials on which Ss received food reward in $\mathrm{j}$.

\section{Method}

The Ss were 36 adult male Long-Evans rats, assigned to four groups of nine. They were fed $2 \mathrm{hr}$. daily, with water always available, and were trained and extinguished at $20 \mathrm{hr}$. of food deprivation. For seven days prior to training they were tamed, habituated to the apparatus, and adapted to the feeding regimen.

A partially reinforced group received a total of 50 training trials in the runway, 25 reward trials interspersed irregularly with 25 nonreward trials (Group 2525). The pattern of reward $(+)$ and nonreward $(-)$ was ,+-+-+--++-++-+---++- , and --+-+++--+ for Trials 1-30. Trials $31-40$ repeated the second of these patterns, and Trials 41-50 repeated the third. Three continuous groups received either 50 reward trials (Group 50-0), 25 reward trials (Group 25-0), or 25 nonreward trials (Group 0-25) during training. Reward consisted of one 195-mg Krummette pellet. Groups 25-25 and 50-0 got twice as many daily training trials as 25-0 and 0-25: two trials on the first day, four on the second and third, and 10 thereafter. Following the last day of training all groups got two days of extinction, 10 trials per day. The average intertrial interval was about $15 \mathrm{~min}$. throughout.

The runway was 4 in. wide, 4.75 in. high, and 54 in. long. It was divided into two sections by a metal floor treadle, $3.5 \mathrm{in}$. wide and $10 \mathrm{in}$. long, pivoted halfway between the two ends of the treadle. The pivot point marked the end of the 40-in. stem section and the beginning of the 14-in. goal section. A food cup was fastened to the end wall of the goal section, just beyond and below a wooden barrier which put the cup out of sight from the stem section. The hinged end wall of the stem section served as S's entrance to the stem. Floor, walls, and treadle were painted midgray, and the ceiling consisted of a transparent Plexiglas panel.

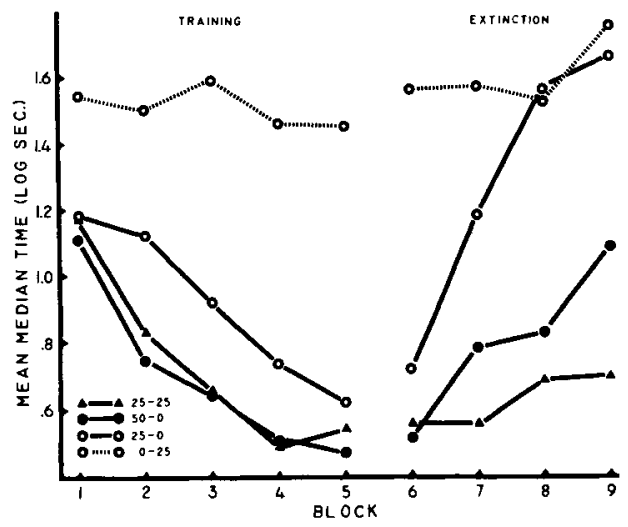

Fig. 1. Time in i (running time). Each training block consists of ten trials for Groups 25-25 and 50-0, and five for Groups 25-0 and 0-25. Each extinction block consists of five trials. 
Running and retrace times were measured automatically by .01-sec. clocks. S was removed from the runway $15 \mathrm{sec}$. after retracing. If $S$ failed to respond within $120 \mathrm{sec}$. , it was nudged into the goal section or stem, and running or retrace time was recorded as $120 \mathrm{sec}$.

\section{Results and Discussion}

Analysis of variance of first-trial data showed that the appropriate groups were adequately matched at the beginning of training. Since most of the statistically significant comparisons described below were significant at $p<.001, p$ values are reported only for the exceptions.

Running time. As training progressed running time decreased significantly for all groups but $0-25$ (see Fig. 1). On the last block of training trials there were no significant differences among 25-25, 50-0, and 25-0.

The extinction data showed a typical partial reinforcement effect, with Group, Trial Block, and their interaction significant sources of variance. Group 25-25 extinguished least rapidly, followed in order by 50-0 and 25-0. Significant differences on the first trial block were entirely attributable to Group 0-25, which ran significantly slower than the other groups combined. On the last trial block $0-25$ and 25-0 did not differ. Combined, they ran significantly slower than 50-0, which in turn ran slower than 25-25 $(\mathrm{p}<.05)$.

Retrace time. Retrace time on reward trials was a function of Group $(p<.025)$ and the Group by Trial Block interaction. Group 50-0 showed a significant decrease as training progressed, while 25-25 and 25-0 showed smaller, insignificant decreases (see Fig. 2).

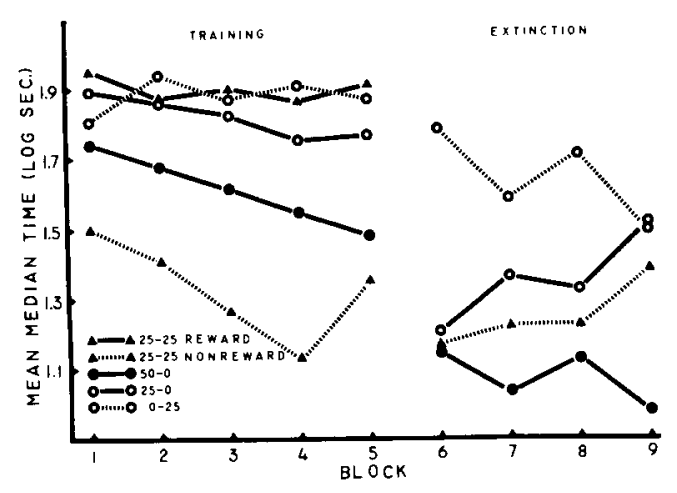

Fig. 2. Time in $\mathbf{j}$ (retrace time). Each training block consists of ten trials for Groups 25-25 and 50-0, and five for Groups 25-0 and 0-25. Each extinction block consists of five trials.
Significant differences on the last trial block were entirely attributable to 50-0, which retraced significantly faster than 25-25 and 25-0 combined.

During training 25-25 retraced significantly faster than $\mathbf{0 - 2 5}$ on nonreward trials, as predicted. Neither Trial Block nor the interaction between Group and Trial Block was a significant source of variance, indicating that the difference between $25-25$ and 0-25 developed and stabilized soon after training began. On every trial block all or nearly all Ss in 25-25 retraced faster on nonreward than reward trials.

The extinction data revealed a significant Group effect $(p<.005)$, and the groups were ordered as predicted: 50-0 retraced fastest, followed by 25-25 and $25-0$ in close order, followed by $0-25$. Other aspects of the extinction data, related to the marginally significant Group by Trial Block interaction $(p<.05)$, were more problematical. According to frustration theory, the predicted ordering should be most likely to occur early in extinction, before the differential reward expectancies established during training have weakened to a common low level. However, significant differences on the first block of trials were entirely attributable to the relatively slow retracing of $0-25$; at that point 50-0 did not differ from 25-25 and 25-0. On the last block of trials 50-0 retraced significantly faster than the other groups combined $(p<.005)$, at which point 25-25 and 25-0 did not differ from 0-25.

In view of the marginal character of the troublesome interaction, serious consideration of its implications for frustration theory had best await additional work with the retrace method-which seems to hold some promise as a means of measuring S's immediate reaction to events in the goal box.

\section{References}

Adelman, H. M., \& Maatsch, J. L. Resistance to extinction as a function of the type of response elicited by frustration. $J$. exp. Psychol., 1955, 50, 61-65.

Amsel, A. The role of frustrative non-reward in non-continuous reward situations. Psychol. Bull., 1958, 55, 102-119.

Brown, J. S. Gradients of approach and avoidance responses and their relation to level of motivation. J. comp. physiol. Psychol. $1948,41,450-465$

Lewis, M. Some nondecremental effects of effort. $J$. comp. physiol. Psychol., 1964, 57, 367-372.

\section{Note}

1. This work was supported in part by Grant MH 11470 from the National Institute of Mental Health, United States Public Health Service, and NSF Undergraduate Research Participation Program Grant GE 4207. I thank James G. Goins and David Volckmann for their assistance. 